\title{
Generalized condensation and the Bogoliubov theory of superfluidity
}

\author{
V.A.Zagrebnov* \\ Université de la Méditerranée and Centre de Physique \\ Théorique-CNRS-Luminy-Case 907, \\ 13288 Marseille, Cedex 09, France
}

Received March 20, 2000

In this paper we scrutinize the Bogoliubov theory of superfluidity on the level of the first Bogoliubov ansatz. This ansatz preconizes the Bogoliubov truncated Hamiltonian as a starting point of this theory. Since this ansatz is based on the hypothesis concerning the Bose-Einstein condensation in zero-mode as well as on its stability with respect to interactions, we present some rigorous results, which cast doubt on this hypothesis.

Key words: Bogoliubov weakly imperfect gas, Bose condensation, generalized condensation

PACS: 05.30.Jp, 02.70.Lq, 03.75.fi, 67.40.Db

\section{Generalized condensation}

Recall that the starting point of the Bogoliubov theory is based on two hypothesis:

(a) there is a Bose-Einstein (BE) condensation in one mode;

(b) this condensation is stable with respect to a weak interaction between particles.

These hypothesis inspired Bogoliubov (see e.g. [1,2]) into his first ansatz: the truncation of the full Hamiltonian

$$
H_{\Lambda}=\sum_{k \in \Lambda^{*}} \varepsilon_{k} a_{k}^{*} a_{k}+\frac{1}{2 V} \sum_{k_{1}, k_{2}, q \in \Lambda^{*}} v(q) a_{k_{1}+q}^{*} a_{k_{2}-q}^{*} a_{k_{2}} a_{k_{1}},
$$

where all sums run over the set $\Lambda^{*}$ defined by

$$
\Lambda^{*}=\left\{k \in \mathbb{R}^{3}: \alpha=1,2,3, k_{\alpha}=\frac{2 \pi n_{\alpha}}{L} \text { et } n_{\alpha}=0, \pm 1, \pm 2, \ldots \ldots\right\},
$$

to the Hamiltonian of the Weakly Imperfect Bose-Gas (WIBG):

$$
H_{\Lambda}^{\mathrm{B}}=T_{\Lambda}+U_{\Lambda}^{\mathrm{D}}+U_{\Lambda}^{\mathrm{ND}},
$$

\footnotetext{
*E-mail: zagrebnov@cpt.univ-mrs.fr
} 
where

$$
\begin{gathered}
T_{\Lambda}=\sum_{k \in \Lambda^{*}} \varepsilon_{k} a_{k}^{*} a_{k} \\
U_{\Lambda}^{\mathrm{D}}=\frac{v(0)}{V} a_{0}^{*} a_{0} \sum_{k \in \Lambda^{*}, k \neq 0} a_{k}^{*} a_{k}+\frac{1}{2 V} \sum_{k \in \Lambda^{*}, k \neq 0} v(k) a_{0}^{*} a_{0}\left(a_{k}^{*} a_{k}+a_{-k}^{*} a_{-k}\right)+\frac{v(0)}{2 V} a_{0}^{*^{2}} a_{0}^{2} \\
U_{\Lambda}^{\mathrm{ND}}=\frac{1}{2 V} \sum_{k \in \Lambda^{*}, k \neq 0} v(k)\left(a_{k}^{*} a_{-k}^{*} a_{0}^{2}+a_{0}^{*^{2}} a_{k} a_{-k}\right) .
\end{gathered}
$$

Here $\varepsilon_{k}=\hbar^{2} k^{2} / 2 m$ is the kinetic energy, and $a_{k}^{\#}=\left\{a_{k}^{*}, a_{k}\right\}$ are usual boson creation and annihilation operators in the one-particle state $\psi_{k}(x)=V^{-\frac{1}{2}} \mathrm{e}^{\mathrm{i} k x}, k \in$ $\Lambda^{*}, x \in \Lambda$; for example, $a_{k}^{*} \equiv a^{*}\left(\psi_{k}\right)=\int_{\Lambda} \mathrm{d} x \psi_{k}(x) a^{*}(x)$, where $a^{\#}(x)$ are bosonfield operators in the Fock space $\mathcal{F}_{\Lambda}$ over $L^{2}(\Lambda)$, see[3].

In this section we scrutinize the (most innocent) hypothesis $(a)$, which concerns essentially the Perfect Bose-Gas (PBG). As it is indicated in [4], the conventional $\mathrm{BE}$ condensation is a subtle matter even for the PBG.

Let instead of the cubic box $\Lambda=L \times L \times L,|\Lambda|=V$, one takes a prism $\Lambda=L_{1} \times L_{2} \times L_{3}$ of the same volume with sides of length $L_{j}=V^{\alpha_{j}}, j=1,2,3$, such that $\alpha_{1} \geqslant \alpha_{2} \geqslant \alpha_{3}>0$ and $\alpha_{1}+\alpha_{2}+\alpha_{3}=1$. We impose periodic boundary conditions on $\partial \Lambda$, and we consider a possibility of conventional BE condensation in this prism. This example is due to Casimir [5] .

Now let us rewrite the equation for the particle density in the form:

$$
\begin{aligned}
& \rho= \frac{1}{V} \frac{1}{\mathrm{e}^{-\beta \mu}-1}+\frac{1}{V} \sum_{k \in\left\{\Lambda^{*}: n_{1} \neq 0, n_{2}=n_{3}=0\right\}} \frac{1}{\mathrm{e}^{\beta\left(\varepsilon_{k}-\mu\right)}-1} \\
&+\frac{1}{V} \sum_{k \in\left\{\Lambda^{*}: n_{j} \neq 0, j=2 \text { or } 3\right\}} \frac{1}{\mathrm{e}^{\beta\left(\varepsilon_{k}-\mu\right)}-1} .
\end{aligned}
$$

For $\rho>\rho_{\mathrm{c}}^{\mathrm{P}}(\theta)$ (the critical density for the PBG) the equation (1.7) gives asymptotic behaviour of the solution $\mu_{\Lambda}^{\mathrm{P}}\left(\theta, \rho>\rho_{\mathrm{c}}^{\mathrm{P}}(\theta)\right)$ and distribution of bosons in the mode $k=0$ and in its vicinity.

(i) Let $\alpha_{1}<1 / 2$. Then for $V \rightarrow \infty$ one gets:

$$
\inf _{k \in \Lambda^{*} \backslash\{0\}} \varepsilon_{k}=O\left(V^{-2 \alpha_{1}}\right) .
$$

This means that

$$
\lim _{\Lambda} \sup _{k \in \Lambda^{*} \backslash\{0\}} \frac{1}{V} \frac{1}{\mathrm{e}^{\beta \varepsilon_{k}}-1}=0
$$

Therefore, the Darboux sum (1.7) for $k \neq 0$ is well-defined and converges to the integral

$$
\rho^{\mathrm{P}}(\beta, 0)=\lim _{\Lambda} \rho_{\Lambda}^{\mathrm{P}}\left(\beta, \mu_{\Lambda}^{\mathrm{P}}\left(\theta, \rho>\rho_{\mathrm{c}}^{\mathrm{P}}(\theta)\right)\right)
$$


Since $\rho>\rho_{\mathrm{c}}^{\mathrm{P}}(\theta)=\rho^{\mathrm{P}}(\beta, 0)$ the equation (1.7) implies that

$$
\mu_{\Lambda}^{\mathrm{P}}\left(\theta, \rho>\rho_{\mathrm{c}}^{\mathrm{P}}(\theta)\right) \simeq \frac{1}{\beta V\left(\rho-\rho_{\mathrm{c}}^{\mathrm{P}}(\theta)\right)}, V \rightarrow \infty
$$

as in the case of cube $\alpha_{1}=\alpha_{2}=\alpha_{3}=1 / 3$. Consequently, one gets a conventional $\mathrm{BE}$ condensation in one mode $k=0$ :

$$
\begin{aligned}
& \lim _{\Lambda} \frac{1}{V}\left\langle N_{k=0}\right\rangle_{T_{\Lambda}}\left(\beta, \mu_{\Lambda}^{\mathrm{P}}\left(\theta, \rho>\rho_{\mathrm{c}}^{\mathrm{P}}(\theta)\right)\right)=\rho_{0}^{\mathrm{P}}(\theta) \equiv \rho-\rho_{\mathrm{c}}^{\mathrm{P}}(\theta)>0, \\
& \lim _{\Lambda} \frac{1}{V}\left\langle N_{k \neq 0}\right\rangle_{T_{\Lambda}}\left(\beta, \mu_{\Lambda}^{\mathrm{P}}\left(\theta, \rho>\rho_{\mathrm{c}}^{\mathrm{P}}(\theta)\right)\right)=0 .
\end{aligned}
$$

(ii) Now let $\alpha_{1}=1 / 2$. Then one gets:

$$
\inf _{k \in \Lambda^{*} \backslash\{0\}} \varepsilon_{k}=\frac{(2 \pi \hbar)^{2}}{2 m} \frac{1}{V}
$$

which corresponds to the mode with $\left(n_{1}=1, n_{2}=0, n_{3}=0\right)$. Since $\alpha_{2}<1 / 2$ and $\alpha_{3}<1 / 2$, we get

$$
\lim _{\Lambda} \sup _{k \in\left\{\Lambda^{*}: n_{j} \neq 0, j=2 \text { or } 3\right\}} \frac{1}{V} \frac{1}{\mathrm{e}^{\beta \varepsilon_{k}}-1}=0 .
$$

Then the Darboux sum corresponding to the last term in (1.7) converges to the integral $\rho^{\mathrm{P}}(\beta, \mu)$ for all $\mu<0$. Since this integral attains its $\sup _{\mu<0} \rho^{\mathrm{P}}(\beta, \mu)=\rho_{\mathrm{c}}^{\mathrm{P}}(\theta)$ for $\mu \rightarrow 0^{-}$, from (1.7) one obtains for $\rho>\rho_{\mathrm{c}}^{\mathrm{P}}(\theta)$ that

$$
\lim _{\Lambda}\left\{\frac{1}{V} \frac{1}{\mathrm{e}^{-\beta \mu_{\Lambda}^{\mathrm{P}}(\theta, \rho)}-1}+\frac{1}{V} \sum_{k \in\left\{\Lambda^{*}: n_{1} \neq 0, n_{2}=n_{3}=0\right\}} \frac{1}{\mathrm{e}^{\beta\left(\varepsilon_{k}-\mu_{\Lambda}^{\mathrm{P}}(\theta, \rho)\right)}-1}\right\}=\rho-\rho_{\mathrm{c}}^{\mathrm{P}}(\theta) .
$$

By virtue of $\alpha_{1}=1 / 2$ the left-hand side of (1.11) implies:

$$
\mu_{\Lambda}^{\mathrm{P}}\left(\theta, \rho>\rho_{\mathrm{c}}^{\mathrm{P}}(\theta)\right)=\frac{A}{V}+o\left(\frac{1}{V}\right), A>0,
$$

for $V \rightarrow \infty$. Since for the asymptotics (1.12) we have

$$
\lim _{\Lambda}\left\{\sum_{k \in\left\{\Lambda^{*}:\left(n_{1}, 0,0\right)\right\}} \frac{1}{\mathrm{e}^{\beta\left(\varepsilon_{k}-\mu_{\Lambda}^{\mathrm{P}}(\theta, \rho)\right)}-1}\right\}=\frac{1}{\beta} \sum_{n_{1}=0, \pm 1, \pm 2, \ldots}\left(\frac{(2 \pi \hbar)^{2}}{2 m} n_{1}^{2}+A\right)^{-1},
$$

the (1.11) implies

$$
\frac{1}{\beta} \sum_{n_{1}=0, \pm 1, \pm 2, \ldots}\left(\frac{(2 \pi \hbar)^{2}}{2 m} n_{1}^{2}+A\right)^{-1}=\rho-\rho_{\mathrm{c}}^{\mathrm{P}}(\theta)
$$


where $A=A(\theta, \rho)$ is a unique root of this equation. Moreover, the limit (1.13) shows that for $\rho>\rho_{\mathrm{c}}^{\mathrm{P}}(\theta)$ :

$$
\begin{aligned}
& \lim _{\Lambda} \frac{1}{V}\left\langle N_{k}\right\rangle_{T_{\Lambda}}\left(\beta, \mu_{\Lambda}^{\mathrm{P}}(\theta, \rho)\right)= \\
& \quad= \begin{cases}\beta^{-1}\left(\frac{(2 \pi \hbar)^{2}}{2 m} n_{1}^{2}+A\right)^{-1}, & k \in\left\{\Lambda^{*}:\left(n_{1}, 0,0\right)\right\}, \\
0 \quad, & k \in\left\{\Lambda^{*}: n_{j} \neq 0, j=2 \text { or } 3\right\} .\end{cases}
\end{aligned}
$$

This means that for the long prism with $\alpha_{1}=1 / 2$ there is macroscopic occupation of an infinite number of low-lying levels $k \in\left\{\Lambda^{*}:\left(n_{1}, 0,0\right)\right\}$ including the zero-mode $k=0$.

(iii) Let $\alpha_{1}>1 / 2$, i.e. we deal with a highly anisotropic prism in direction $j=1$. Then we have

$$
\inf _{k \in \Lambda^{*} \backslash\{0\}} \varepsilon_{k}=\frac{(2 \pi \hbar)^{2}}{2 m} \frac{1}{V^{2 \alpha_{1}}}, 2 \alpha_{1}>1,
$$

which corresponds to the mode with $\left(n_{1}=1, n_{2}=0, n_{3}=0\right)$. Since for any $\mu<0$ the right-hand side of (1.7) converges to the integral $\rho^{\mathrm{P}}(\beta, \mu)$ monotonously increasing up to $\rho_{\mathrm{c}}^{\mathrm{P}}(\theta)$, when $\mu \rightarrow 0^{-}$, the solution $\mu_{\Lambda}^{\mathrm{P}}\left(\theta, \rho>\rho_{\mathrm{c}}^{\mathrm{P}}(\theta)\right)$ of $(1.7)$ has to have the asymptotics :

$$
\mu_{\Lambda}^{\mathrm{P}}\left(\theta, \rho>\rho_{\mathrm{c}}^{\mathrm{P}}(\theta)\right)=\frac{B}{V^{\delta}}+o\left(\frac{1}{V^{\delta}}\right), B<0, \delta>0,
$$

for $V \rightarrow \infty$. To calculate $B$ and $\delta$, notice that the first two terms in (1.7) may be represented as

$$
\begin{gathered}
\frac{1}{V} \sum_{k \in\left\{\Lambda^{*}:\left(n_{1}, 0,0\right)\right\}} \frac{1}{\mathrm{e}^{\beta\left(\varepsilon_{k}-\mu_{\Lambda}^{\mathrm{P}}(\theta, \rho)\right)}-1}=\frac{1}{V} \sum_{k \in\left\{\Lambda^{*}:\left(n_{1}, 0,0\right)\right\}} \sum_{s=1}^{+\infty} \mathrm{e}^{-s \beta\left(\varepsilon_{k}-\mu_{\Lambda}^{\mathrm{P}}(\theta, \rho)\right)} \\
=\frac{1}{V} \sum_{s=1}^{+\infty} \mathrm{e}^{s \beta \mu_{\Lambda}^{\mathrm{P}}(\theta, \rho)} \sum_{n_{1}=0, \pm 1, \pm 2, \ldots} \mathrm{e}^{-s \beta\left(\frac{(2 \pi \hbar)^{2}}{2 m} \frac{n_{1}^{2}}{V^{2 \alpha_{1}}}\right)} .
\end{gathered}
$$

Notice that

$$
\sum_{n_{1}=0, \pm 1, \pm 2, \ldots} \mathrm{e}^{-\pi \lambda n_{1}^{2}}=\frac{1}{\sqrt{\lambda}} \sum_{\xi=0, \pm 1, \pm 2, \ldots} \mathrm{e}^{-\left(\pi \xi^{2} / \lambda\right)}
$$

where $\lambda=\beta \frac{2 \pi \hbar^{2}}{m} s V^{-2 \alpha_{1}}$. Taking into account (1.17)-(1.19) we find that only the term with $\xi=0$ is important for (1.18) when $V \rightarrow \infty$ :

$$
\rho-\rho_{\mathrm{c}}^{\mathrm{P}}(\theta)=\lim _{\Lambda}\left\{\left(\frac{2 \pi \hbar^{2}}{m} \beta\right)^{-1 / 2}\left\{\frac{V^{\alpha_{1}-1}}{V^{\delta / 2}} \cdot V^{\delta}\right\} \frac{1}{V^{\delta}}\left\{\sum_{s=1}^{+\infty} \mathrm{e}^{\beta B\left(s / V^{\delta}\right)}\left(\frac{s}{V^{\delta}}\right)^{-1 / 2}\right\}\right\} .
$$

The limit in (1.20) is nontrivial only for

$$
\delta=2\left(1-\alpha_{1}\right) .
$$


Then one gets

$$
0<\rho-\rho_{\mathrm{c}}^{\mathrm{P}}(\theta)=\left(\frac{2 \pi \hbar^{2}}{m}\right)^{-1 / 2} \beta^{-1 / 2} \int_{0}^{+\infty} \mathrm{d} \xi \mathrm{e}^{\beta B \xi} \xi^{-1 / 2}
$$

where $B=B\left(\theta=\beta^{-1}, \rho\right)<0$ is the unique root of equation (1.22), or

$$
\rho-\rho_{\mathrm{c}}^{\mathrm{P}}(\theta)=\left(\frac{m}{2 \hbar^{2}}\right)^{1 / 2} \frac{\theta}{\sqrt{|B(\theta, \rho)|}},
$$

since

$$
I=\int_{0}^{+\infty} \mathrm{d} y \mathrm{e}^{-y} y^{-1 / 2}=\sqrt{\pi}
$$

By virtue of $(1.17),(1.21)$ and $(1.23)$ we get that for $\rho>\rho_{\mathrm{c}}^{\mathrm{P}}(\theta)$ and $V \rightarrow \infty$ :

$$
\begin{aligned}
& \varepsilon_{k}-\mu_{\Lambda}^{\mathrm{P}}(\theta, \rho) \simeq \\
& \simeq\left\{\begin{array}{l}
\frac{\hbar^{2}}{2 m}\left(\frac{2 \pi n_{1}}{V^{\alpha_{1}}}\right)^{2}+\frac{m / 2 \hbar^{2}}{\beta^{2}\left(\rho-\rho_{\mathrm{c}}^{\mathrm{P}}(\theta)\right)^{2} V^{2\left(1-\alpha_{1}\right)}}, k \in\left\{\Lambda^{*}:\left(n_{1}, 0,0\right)\right\}, \\
\frac{\hbar^{2}}{2 m}\left[\left(\frac{2 \pi n_{2}}{V^{\alpha_{2}}}\right)^{2}+\left(\frac{2 \pi n_{3}}{V^{\alpha_{3}}}\right)^{2}\right]+\frac{m / 2 \hbar^{2}}{\beta^{2}\left(\rho-\rho_{\mathrm{c}}^{\mathrm{P}}(\theta)\right)^{2} V^{2\left(1-\alpha_{1}\right)}}, k \in\left\{\Lambda^{*}: n_{j=2} \text { or } 3 \neq 0\right\} .
\end{array}\right.
\end{aligned}
$$

Since $\alpha_{1}>1 / 2$ and $\alpha_{1}+\alpha_{2}+\alpha_{3}=1$, the asymptotics (1.24) imply

$$
\lim _{\Lambda} \frac{1}{V}\left\langle N_{k}\right\rangle_{T_{\Lambda}}\left(\beta, \mu_{\Lambda}^{\mathrm{P}}\left(\theta, \rho>\rho_{\mathrm{c}}^{\mathrm{P}}(\theta)\right)\right)=0, k \in \Lambda^{*}
$$

while

$$
\lim _{\Lambda} \frac{1}{V^{2\left(1-\alpha_{1}\right)}}\left\langle N_{k}\right\rangle_{T_{\Lambda}}\left(\beta, \mu_{\Lambda}^{\mathrm{P}}\left(\theta, \rho>\rho_{\mathrm{c}}^{\mathrm{P}}(\theta)\right)\right)=\frac{m / 2 \hbar^{2}}{\beta^{2}\left(\rho-\rho_{\mathrm{c}}^{\mathrm{P}}(\theta)\right)^{2}}, k \in\left\{\Lambda^{*}:\left(n_{1}, 0,0\right)\right\},
$$

and (cf. (1.7), (1.18), (1.20))

$$
\rho_{0}^{\mathrm{P}}(\theta)=\rho-\rho_{\mathrm{c}}^{\mathrm{P}}(\theta)=\lim _{\Lambda} \frac{1}{V} \sum_{k \in\left\{\Lambda^{*}:\left(n_{1}, 0,0\right)\right\}}\left\langle N_{k}\right\rangle_{T_{\Lambda}}\left(\beta, \mu_{\Lambda}^{\mathrm{P}}(\theta, \rho)\right)>0 .
$$

This means that for the extremely long prism with $\alpha_{1}>1 / 2$ for $\rho>\rho_{\mathrm{c}}^{\mathrm{P}}(\theta)$ there is conventional $\mathrm{BE}$ condensation with density $\rho_{0}^{\mathrm{P}}(\theta)>0$, whereas there are no macroscopically occupied levels in $\Lambda^{*}$.

Cases $\alpha_{1}=1 / 2$ and $\alpha_{1}>1 / 2$ are provided examples of generalized condensation in the ]irardeau sense [6]. This gives a motivation for the following van den BergLewis-Pulè's classification of the generalized BE condensation $[4,7,8]$ :

- the condensation is called the type $I$ when a finite number of single-particle levels are macroscopically occupied; 
- it is of type $I I$ when an infinite number of the levels are macroscopically occupied;

- it is called the type III, or the non-extensive condensation, when none of the levels is macroscopically occupied, whereas one has:

$$
\lim _{\delta \rightarrow 0^{+}} \lim _{\Lambda} \frac{1}{V} \sum_{\left\{k \in \Lambda^{*}, 0 \leqslant\|k\| \leqslant \delta\right\}}\left\langle N_{k}\right\rangle=\rho-\rho_{\mathrm{c}}(\theta) .
$$

Remark 1 Returning back to the Bogoliubov theory we have to recognize that the hypothesis (a) about the one-mode BE condensation looses its grip if we pass to the PBG in a long prism. The latter is not an exotic situation if one considers superfluidity of extremely thin films of ${ }^{4} \mathrm{He}$, see $[9,10]$.

\section{The model}

In $[11,12]$ it was shown that the type III condensation can be caused in the PBG by a weak external field or by a specific choice of the boundary conditions and geometry $[7,13]$. But recently other examples of this non-extensive $\mathrm{BE}$ condensation were discovered for bosons in an isotropic box $\Lambda$ with repulsive interaction which spreads out the conventional BE condensation of the type I into the type III, [14-16].

To avoid the influence of the shape on the BE condensation, let us take the cubic box $\Lambda=L \times L \times L$. Imposing the periodic boundary conditions on $\partial \Lambda$, we consider a simple interacting boson system with the Hamiltonian:

$$
H_{\Lambda}^{\mathrm{I}}=\sum_{k \in \Lambda^{*}} \varepsilon_{k} a_{k}^{*} a_{k}+\frac{1}{2 V} \sum_{k \in \Lambda^{*}} v(0) a_{k}^{*} a_{k}^{*} a_{k} a_{k}, v(0)>0 .
$$

Interaction in (2.1) results from a "severe" truncation of the full interaction $H_{\Lambda}$. Hereinafter we shall demonstrate that even this "small" part of the total interaction can drastically change the distribution of condensed particles spreading out the BE condensation in the one mode $k=0$ into non-extensive $\mathrm{BE}$ condensation of the type III.

First we show that the interaction in (2.1) is so gentle that it even does not modify the pressure of the perfect system.

Theorem 1 For the model (2.1) the thermodynamic limit of the pressure coincides with the pressure of the $P B G$ :

$$
\lim _{\Lambda} p_{\Lambda}\left[H_{\Lambda}^{\mathrm{I}}\right]=\lim _{\Lambda} p_{\Lambda}\left[T_{\Lambda}\right]=p^{\mathrm{P}}(\beta, \mu) .
$$

Proof. Notice that $a_{k}^{*} a_{k}^{*} a_{k} a_{k}=N_{k}^{2}-N_{k}$. Therefore, the grand partition function for the model (2.1) takes the form :

$$
\Xi_{\Lambda}^{\mathrm{I}}(\beta, \mu)=\operatorname{Tr}_{\mathcal{F}_{\mathrm{B}}} \mathrm{e}^{-\beta\left(H_{\Lambda}^{\mathrm{I}}-\mu N_{\Lambda}\right)}=\prod_{k \in \Lambda^{*}} \sum_{n_{k}=0}^{+\infty} \mathrm{e}^{-\beta\left[\left(\varepsilon_{k}-\mu\right) n_{k}+\frac{v(0)}{2 V}\left(n_{k}^{2}-n_{k}\right)\right]}
$$


which converges for all $\mu \in \mathbb{R}^{1}$. Then for the pressure in the finite volume we have the estimates:

$$
p_{\Lambda}\left[T_{\Lambda}\right] \geqslant p_{\Lambda}\left[H_{\Lambda}^{\mathrm{I}}\right] \geqslant \frac{1}{\beta V} \sum_{k \in \Lambda^{*}} \ln \sum_{n_{k}=0}^{[\ln V]} \mathrm{e}^{-\beta\left[\left(\varepsilon_{k}-\mu\right) n_{k}+\frac{v(0)}{2 V}\left(n_{k}^{2}-n_{k}\right)\right]} .
$$

By virtue of $n_{k}^{2}-n_{k} \leqslant[\ln V]^{2}$ (here $[x]$ denotes the integer part of $x$ ) one gets:

$$
\begin{aligned}
& \sum_{n_{k}=0}^{[\ln V]} \mathrm{e}^{-\beta\left[\left(\varepsilon_{k}-\mu\right) n_{k}+\frac{v(0)}{2 V}\left(n_{k}^{2}-n_{k}\right)\right]} \geqslant \\
& \quad \geqslant \sum_{n_{k}=0}^{[\ln V]} \mathrm{e}^{-\beta\left[\left(\varepsilon_{k}-\mu\right) n_{k}+\frac{v(0)}{2 V}[\ln V]^{2}\right]}=\mathrm{e}^{-\beta \frac{v(0)}{2 V}[\ln V]^{2}}\left\{\frac{1-\mathrm{e}^{-\beta\left(\varepsilon_{k}-\mu\right)([\ln V]-1)}}{1-\mathrm{e}^{-\beta\left(\varepsilon_{k}-\mu\right)}}\right\} .
\end{aligned}
$$

For $\mu<0$ the left-hand side of (2.5) converges (uniformly in $k \in \Lambda^{*}$ ) to $\left(1-\mathrm{e}^{-\beta\left(\varepsilon_{k}-\mu\right)}\right)^{-1}$ when $V \rightarrow \infty$. Hence, in the thermodynamic limit, the lower estimate in (2.4) coincides with the upper estimate:

$$
\begin{aligned}
\lim _{\Lambda} \frac{1}{\beta V} \sum_{k \in \Lambda^{*}} \ln \left\{\mathrm{e}^{-\beta \frac{v(0)}{2 V}[\ln V]^{2}} \frac{1-\mathrm{e}^{-\beta\left(\varepsilon_{k}-\mu\right)([\ln V]-1)}}{\left.1-\mathrm{e}^{-\beta\left(\varepsilon_{k}-\mu\right)}\right\}=}\right. \\
=\lim _{\Lambda} \frac{1}{\beta V} \sum_{k \in \Lambda^{*}} \ln \left(1-\mathrm{e}^{-\beta\left(\varepsilon_{k}-\mu\right)}\right)^{-1} \\
=\lim _{\Lambda} p_{\Lambda}\left[T_{\Lambda}\right]
\end{aligned}
$$

which proves the equality (2.2). Since $\lim _{\mu \rightarrow 0^{-}} \lim _{\Lambda} p_{\Lambda}\left[T_{\Lambda}\right]$ is bounded, one extends $p^{\mathrm{P}}(\beta, \mu)$ to $\mu=0$ by continuity.

As usual, the total density of particles for the model (2.1) is defined in the grand-canonical ensemble for a finite volume $V$ by :

$$
\rho_{\Lambda}(\beta, \mu)=\left\langle\frac{N_{\Lambda}}{V}\right\rangle_{H_{\Lambda}^{\mathrm{I}}}(\beta, \mu)=\frac{1}{V} \sum_{k \in \Lambda^{*}}\left\langle N_{k}\right\rangle_{H_{\Lambda}^{\mathrm{I}}}(\beta, \mu)=\partial_{\mu} p_{\Lambda}\left[H_{\Lambda}^{\mathrm{I}}\right] .
$$

Corollary 2 Due to $v(0)>0$ the pressure $p_{\Lambda}\left[H_{\Lambda}^{\mathrm{I}}\right]$ is defined for all chemical potentials $\mu \in \mathbb{R}^{1}$, see (2.3). Moreover it is obviously a convex function of this parameter. The limit function $p^{\mathrm{P}}(\beta, \mu)$ is defined only for $\mu \leqslant 0$ and is also a convex function, but only for $\mu<0$. Since (2.2) is the statement regarding the convergence of convex functions of $\mu \in(-\infty, 0)$, by the Griffiths lemma we get convergence of the corresponding derivatives (2.6)):

$$
\lim _{\Lambda} \rho_{\Lambda}^{\mathrm{I}}(\beta, \mu)=\lim _{\Lambda} \partial_{\mu} p_{\Lambda}\left[H_{\Lambda}^{\mathrm{I}}\right]=\partial_{\mu} \lim _{\Lambda} p_{\Lambda}\left[H_{\Lambda}^{\mathrm{I}}\right]=\partial_{\mu} p^{\mathrm{P}}(\beta, \mu)=\rho^{\mathrm{P}}(\beta, \mu),
$$

on the very same interval $\mu \in(-\infty, 0)$. So, in the thermodynamic limit the total particle density $\rho^{\mathrm{I}}(\beta, \mu)$ of the model (2.1) coincides with $\rho^{\mathrm{P}}(\beta, \mu)$. Since $\lim _{\mu \rightarrow 0^{-}} \rho^{\mathrm{P}}(\beta, \mu)$ is bounded for $d>2$, one defines $\rho^{\mathrm{I}}(\beta, \mu=0)=\rho^{\mathrm{P}}(\beta, \mu=0)$ by continuity. 
From this corollary one deduces that, as in the case of the $\mathrm{PBG}, \rho^{\mathrm{I}}(\beta, \mu)$ attains its maximum $\rho_{\mathrm{c}}^{\mathrm{I}}(\theta)=\lim _{\mu \rightarrow 0^{-}} \rho^{\mathrm{I}}(\beta, \mu)=\rho_{\mathrm{c}}^{\mathrm{P}}(\theta)$ at $\mu=0$, i.e. for densities $\rho>\rho_{\mathrm{c}}^{\mathrm{P}}(\theta)$ one must have a BE condensation. On the other hand, for the expectation of the ground-state occupation number for $\mu=0$ we get from (2.1):

$$
\left\langle\frac{N_{0}}{\sqrt{V}}\right\rangle_{H_{\Lambda}^{\mathrm{I}}}(\beta, \mu=0)=\frac{\frac{1}{\sqrt{V}} \sum_{n_{0}=0}^{+\infty} \frac{n_{0}}{\sqrt{V}} \mathrm{e}^{-\beta \frac{v(0)}{2}\left(\frac{n_{0}}{\sqrt{V}}\right)^{2}}}{\frac{1}{\sqrt{V}} \sum_{n_{0}=0}^{+\infty} \mathrm{e}^{-\beta \frac{v(0)}{2}\left(\frac{n_{0}}{\sqrt{V}}\right)^{2}} .}
$$

Since the numerator and the denominator in the right-hand side of (2.7) are nothing but Darboux sums for the corresponding integrals in the limit $V \rightarrow \infty$, we obtain that

$$
\lim _{\Lambda} \frac{1}{\sqrt{V}}\left\langle N_{0}\right\rangle_{H_{\Lambda}^{\mathrm{I}}}(\beta, \mu=0)=\frac{\int \mathrm{d} x \cdot x \cdot \mathrm{e}^{-\beta \frac{v(0)}{2} x^{2}}}{\int \mathrm{d} x \cdot \mathrm{e}^{-\beta \frac{v(0)}{2} x^{2}}}=\frac{1}{2} \sqrt{\frac{2 \theta}{v(0)}} .
$$

This means that there is no macroscopic occupation of the mode $k=0$ because $\left\langle N_{0}\right\rangle_{H_{\Lambda}^{\mathrm{I}}}(\beta, \mu=0)$ growth only as $V^{1 / 2}$ for $V \rightarrow \infty$.

Theorem 3 For any parameters $(\beta, \mu \leqslant 0)$ one has

$$
\lim _{\Lambda} \frac{1}{V}\left\langle N_{k}\right\rangle_{H_{\Lambda}^{\mathrm{I}}}(\beta, \mu)=0, \forall k \in \Lambda^{*}
$$

i.e. there is no macroscopic occupation of any mode $k \in \Lambda^{*}$.

Proof. By the Bogoliubov convexity inequality for the pressure (see e.g. $[17,18]$ ), we get

$$
\frac{v(0)}{2 V^{2}}\left\langle\sum_{k \in \Lambda^{*}}\left(N_{k}^{2}-N_{k}\right)\right\rangle_{H_{\Lambda}^{\mathrm{I}}} \leqslant p_{\Lambda}\left[T_{\Lambda}\right]-p_{\Lambda}\left[H_{\Lambda}^{\mathrm{I}}\right] \leqslant \frac{v(0)}{2 V^{2}}\left\langle\sum_{k \in \Lambda^{*}}\left(N_{k}^{2}-N_{k}\right)\right\rangle_{T_{\Lambda}} .
$$

By virtue of $v(0)>0, N_{k}^{2}-N_{k} \geqslant\left(N_{k}-I\right)^{2}$ and theorem 1 , one has

$$
\lim _{\Lambda} \frac{1}{V^{2}} \sum_{k \in \Lambda^{*}}\left\langle\left(N_{k}-I\right)^{2}\right\rangle_{H_{\Lambda}^{\mathrm{I}}}(\beta, \mu \leqslant 0)=0
$$

Notice that by the Cauchy-Schwarz inequality for the Gibbs state we get

$$
\left\langle N_{k}-I\right\rangle_{H_{\Lambda}^{\mathrm{I}}}^{2} \leqslant\left\langle\left(N_{k}-I\right)^{2}\right\rangle_{H_{\Lambda}^{\mathrm{I}}}
$$

Then

$$
0 \leqslant\left(\frac{1}{V}\left\langle N_{k}-I\right\rangle_{H_{\Lambda}^{\mathrm{I}}}\right)^{2} \leqslant \frac{1}{V^{2}}\left\langle\left(N_{k}-I\right)^{2}\right\rangle_{H_{\Lambda}^{\mathrm{I}}} \leqslant \frac{1}{V^{2}} \sum_{q \in \Lambda^{*}}\left\langle\left(N_{q}-I\right)^{2}\right\rangle_{H_{\Lambda}^{\mathrm{I}}} .
$$


The estimates (2.10) and (2.11) give the assertion (2.9).

Resuming corollary 2 and theorem 3 we must admit that the model (2.1) gives an example of non-extensive (i.e. the type III) BE condensation $\rho_{0}(\theta)=\rho-\rho_{\mathrm{c}}(\theta)$. Namely, for $\rho>\rho_{\mathrm{c}}^{\mathrm{I}}(\theta)=\rho_{\mathrm{c}}^{\mathrm{P}}(\theta)$ one has

$$
\lim _{\Lambda} \frac{1}{V}\left\langle N_{k}\right\rangle_{H_{\Lambda}^{\mathrm{I}}}\left(\beta, \mu_{\Lambda}\left(\theta, \rho>\rho_{\mathrm{c}}(\theta)\right)\right)=0, k \in \Lambda^{*}
$$

and at the same time

$$
\begin{aligned}
\rho & =\lim _{\Lambda} \rho_{\Lambda}\left(\beta, \mu_{\Lambda}\left(\theta, \rho>\rho_{\mathrm{c}}(\theta)\right)\right) \\
& =\rho_{\mathrm{c}}(\theta)+\lim _{\delta \rightarrow 0^{+}} \lim _{\Lambda} \frac{1}{V} \sum_{\left\{k \in \Lambda^{*}: 0 \leqslant\|k\| \leqslant \delta\right\}}\left\langle N_{k}\right\rangle_{H_{\Lambda}^{\mathrm{I}}}\left(\beta, \mu_{\Lambda}\left(\theta, \rho>\rho_{\mathrm{c}}(\theta)\right)\right) .
\end{aligned}
$$

Remark 2 Returning back to the Bogoliubov theory we again recognize that there is a problem with the hypothesis $(a)$ and $(b)$ regarding the one-mode (i.e. type I) BE condensation. Even if it exists in $P B G$, a very gentle interaction (e.g. (2.1)), which does not change the pressure of the $P B G$, may drastically change the asymptotics of the occupation number expectations, cf. (2.8). In the model (2.1) the interaction spreads out the type I condensation of the PBG into the type III BE condensation, which means that the Bogoliubov hypothesis (b) is à priori doubtful.

\section{Conclusion}

The perturbation of the model (2.1) is a function of only occupation-number operators, i.e. this interaction commutes with the kinetic-energy operator. This class of interactions is called diagonal. In the series of papers [19-23], van den Berg, Dorlas, Lewis and Pulè gave an exhausting analysis of a hole class of diagonal models (including the exact solution of the Huang-Yang-Luttinger model ), showing how subtle and multiform is the BE condensation in presence of this type of interaction. Since the interaction is diagonal, their analysis is based on a refined Large Deviation Principle of the classical probability. For another approach to the Huang-YangLuttinger model see [24].

If one accepts the first Bogoliubov ansatz, one is faced with a nondiagonal interaction $U_{\Lambda}^{\mathrm{ND}}$ in (1.3), which does not commute with $T_{\Lambda}$. Therefore, the existence of the $k=0$ mode BE condensation in the Bogoliubov WIBG $H_{\Lambda}^{\mathrm{B}}$ is screaming for a rigorous analysis, see $[25,26]$

Returning back to the theory of superfluidity we have to avow that the discussion above is rather far away from real systems. In the recent paper by Vakarchuk [27] one can find a discussion of the difficulties and of the methods going beyond the Bogoliubov theory of superfluidity. Developing some ideas by Bogoliubov, Zubarev and Yukhnovskii regarding the method of collective variables, he obtained new convincing results (see references quoted in [27]) to advance in this challenging unsolved problem of theoretical and mathematical physics. 
The paper is dedicated to Professor Academician Yukhnovskii. I am very thankful to the journal of Condensed Matter Physics for the invitation to write this paper and for an occasion to express in this way my sincere respects to Igor Rafailovich.

\section{References}

1. Bogoliubov N.N. About the theory of superfluidity. // Izv. Akad. Nauk USSR, 1947, vol. 11, p. 77-90.

2. Bogoliubov N.N. Lectures on Quantum Statistics. - In: Collection of papers. vol. 2. Kiev, Naukova Dumka, 1970, p. 287-493.

3. Petrina D.Ya. Mathematical Foundations of Quantum Statistical Mechanics. Kluwer Academic Publishers, 1995.

4. van den Berg M., Lewis J.T. On generalized condensation in the free boson gas. // Physica A, 1982, vol. 110, p. 550-564.

5. Casimir H.B.G. On Bose-Einstein condensation. - In: Fundamental Problems in Statistical Mechanics III, ed. E.G.D. Cohen. Amsterdam, North-Holland Publishing Company, 1968, p. 188-196.

6. Girardeau M. Relationship between systems of impenetrable bosons and fermions in one dimension. // J. Math. Phys., 1960, vol. 1, p. 516-523.

7. van den Berg M. On boson condensation into an infinite number of low-lying levels. // J. Math. Phys., 1982, vol. 23, p. 1159-1161.

8. van den Berg M., Lewis J.T., Pulè J.V. A general theory of Bose-Einstein condensation. // Helv. Phys. Acta., 1986, vol. 59, p. 1271-128.

9. Khalatnikov I.M. An Introduction to the Theory of Superfluidity. New York, Benjamin, 1965.

10. Wilks J. An Introduction to Liquid Helium. Oxford, Claredon, 1970.

11. van den Berg M., Lewis J.T. On the free boson gas in a weak external potential. // Commun. Math. Phys., 1981, vol. 81, p. 475-494.

12. Pulè J.V. The free boson gas in a weak external potential. // J. Math. Phys., 1983, vol. 24, p. 138-142.

13. van den Berg M. On condensation in the free-bosons gas and the spectrum of the Laplacian. // J. Stat. Phys., 1983, vol. 31, p. 623-637.

14. Michoel T., Verbeure A. Nonextensive Bose-Einstein condensation model. // J. Math. Phys., 1999, vol. 40, p. 1268-1279.

15. Bru J.-B., Zagrebnov V.A. Exactly soluble model with two kinds of Bose-Einstein condensations. // Physica A, 1999, vol. 268, p. 309-325.

16. Bru J.-B., Zagrebnov V.A. A model with coexistence of two kinds of Bose condensations. // J. Phys. A: Math. Gen., 2000, vol. 33, p. 449-464.

17. Bogoliubov N.N. (Jr) A Method of Studying Model Hamiltonians. Oxford, Pergamon, 1977.

18. Bogoliubov N.N. (Jr), Brankov J.G., Zagrebnov V.A., Kurbatov A.M., Tonchev N.S. The Approximating Hamiltonian method in Statistical Physics. Sofia, Publ. Bulgarian Acad. Sciences, 1981.

19. van den Berg M., Lewis J.T., Pulè J.V. The large deviation principle and some models of an interacting boson gas. // Commun. Math. Phys., 1988, vol. 118, p. 61-85.

20. van den Berg M., Dorlas T.C., Lewis J.T., Pulè J.V. A perturbed meanfield model of an interacting boson gas and the large deviation principle. // Commun. Math. Phys., 
1990, vol. 127 , p. 41-69.

21. van den Berg M., Dorlas T.C., Lewis J.T., Pulè J.V. The pressure in the Huang-YangLuttinger model of an interacting boson gas. // Commun. Math. Phys., 1990, vol. 128, p. 231-245.

22. Dorlas T.C., Lewis J.T., Pulè J.V. The full diagonal model of a Bose gas. // Commun. Math. Phys., 1993, vol. 156, p. 37-65.

23. Dorlas T.C., Lewis J.T., Pulè J.V. Condensation in some perturbed meanfield models of a Bose gas. // Helv. Phys. Acta., 1991, vol. 64, p. 1200-1224.

24. Petrina D.Ya., Gredzhuk V.I. On two models of interacting Bose gas: Bogolyubov model of superfluidity and Huang-Yang-Lattinger model. // Mat. Fizika. Anal. Geometr., 1996, vol. 3, p. 370-405.

25. Bru J.-B., Zagrebnov V.A. Exact solution of the Bogoliubov Hamiltonian for Weakly Imperfect Bose gas. // J. Phys. A: Math.Gen., 1998, vol. 31, p. 9377-9404.

26. Zagrebnov V.A. The Bogoliubov weakly imperfect Bose-gas. // J. Physical Studies, 1999, vol. 3, p. 239-251.

27. Vakarchuk I.O. Statistical operator of a system of identical interacting particles in coordinate representation. // J. Physical Studies, 1996, vol. 1, p. 25-38.

\section{Узагальнена конденсація і теорія надплинності Боголюбова}

\section{В.А.Загребнов}

Середземноморський університет і центр теоретичної фізики, 13288 Марсель, Франція

Отримано 20 березня 2000 р.

У цій статті ми детально досліджуємо теорію надплинності Боголюбова на рівні першого анзацу Боголюбова. Цей анзац стверджує обрізаний гамільтоніан Боголюбова як стартову точку цієї теорії. Оскільки цей анзац ґрунтується на гіпотезі стосовно Бозе-Ейнштейна конденсації в нульовій моді і на її стабільності по відношенню до взаємодії, ми представляємо строгі результати, які піддають сумніву цю гіпотезу.

Ключові слова: слабо неідеальний газ Боголюбова, Бозе конденсація, узагальнена конденсація

PACS: 05.30.Jp, 02.70.Lq, 03.75.fi, 67.40.Db 
